\title{
Hiperplasia fibrosa focal: lesión benigna de la mucosa oral. Revisión de la literatura.
}

Focal fibrous hyperplasia: benign lesion of the oral mucosa. Literature review.

\author{
María del Carmen Navas-Aparicio ${ }^{1,2, a}$, Patricia Hernández-Rivera ${ }^{1, b}$
}

\section{RESUMEN}

La hiperplasia fibrosa focal es una patología común en cavidad bucal, la cual se caracteriza por ser una lesión hiperplásica inflamatoria reactiva, en respuesta ante un trauma crónico.

Aunque la hiperplasia fibrosa focal es una lesión común, frecuentemente no adquiere la importancia correspondiente por los profesionales, y es transcendental poseer los conocimientos claros ante el diagnóstico y manejo adecuado de esta patología.

PALABRAS CLAVE: fibroma, patología, mucosa bucal, manifestaciones bucales, diagnóstico.

\begin{abstract}
Focal fibrous hyperplasia is a common pathology in the oral cavity, which is characterized by a reactive inflammatory hyperplastic lesion in response to chronic trauma.

Although focal fibrous hyperplasia is a common lesion, it frequently does not acquire the corresponding importance by professionals, and it is essential to have a clear understanding of the proper diagnosis and management of this pathology.
\end{abstract}

KEY WORDS: fibroma, pathology, mouth mucosa, oral manifestations, diagnosis

\footnotetext{
Universidad de Costa Rica. San José, Costa Rica

Hospital Nacional de Niños "Dr. Carlos Sáenz Herrera", Caja Costarricense de Seguro Social. San José, Costa Rica

Maestría en Odontopediatría, Especialista en Cirugía Oral y Maxilofacial

Maestría en Administración Educativa.
} 


\section{INTRODUCCIÓN}

Las lesiones de la mucosa oral se pueden observar como una alteración anatómica en el color, la superficie y/ o su integridad; aunque algunas son benignas y requieren de tratamientos poco invasivos, otras pueden ser una patología maligna. Estas lesiones tienen diferentes etiologías, sean de origen bacteriano, viral, fúngico, o por trauma local o irritacional, enfermedades sistémicas o relacionadas con el consumo excesivo de tabaco y/o alcohol (1).

En cuanto a la lesión reactiva local es la patología de tejido conectivo fibroso, en la cavidad oral, más común, debido al trauma frecuente al que están expuestos estos tejidos; siendo su etiología los irritantes locales, lo que estimula una reparación exagerada del tejido $(2,3,4,5,6,7)$; su tamaño varía dependiendo de la reacción inflamatoria y la respuesta al sanado (7).

Estetipodelesiones se presentan, según la literatura, en un rango de 6,7\% a 48\%; esta variación tan amplia en la prevalencia de las lesiones reactivas locales en múltiples estudios se debe a distintas variables como, diversidad en los estilos de vida, zonas geográficas, diferencia en la nomenclatura aplicada a estas lesiones, características étnicas, y la población de estudio involucrada. Además, se enfatiza que muchas de estas lesiones han sido diagnosticadas clínicamente, pero sin realización de la biopsia respectiva, lo cual puede afectar el diagnóstico apropiado y su tratamiento (8). Importante es destacar, que la presentación clínica y epidemiológica de la mayoría de las lesiones con crecimiento no neoplásico en la cavidad oral son muy similares, por lo que la identificación de estas obedece al estudio histológico (9).

Entre estas lesiones sobresalen, principalmente, la hiperplasia fibrosa focal $(2,3,7,8,10,11)$, el granuloma piógeno, el granuloma de células gigantes periféricas y el fibroma osificante periférico $(2,3,7,8,12)$; aunque en la literatura enumeran otras lesiones reactivas locales, como por ejemplo, el fibroma de células gigantes (8), la hiperplasia fibrosa inflamatoria (12), el épulis fibroso y épulis del embarazo (8), desconociendo que algunas de estas últimas pueden ser las mismas lesiones con nomenclatura diferente.
La hiperplasia fibrosa focal es una lesión común en cavidad oral $(5,7,9,11,13)$. Actualmente existe escasez de estudios amplios relacionados a esta lesión (11), por lo que se realiza esta revisión para especificar sus características clínicas, histológicas, epidemiológicas y conducta de tratamiento, así como su diagnóstico diferencial, con el fin de reforzar los conocimientos, la destreza y habilidad del profesional del área de la salud para identificar aquellas condiciones que no son normales en un paciente y que requieran un diagnóstico y tratamiento pertinente.

\section{Características clínicas}

La hiperplasia fibrosa focal es una lesión reactiva inflamatoria hiperplásica $(5,11,14,15)$, de tejido conectivo $(7,10,11)$, que prolifera de manera exagerada al trauma crónico $(5,7,9,10,11,14,15,16)$. También se le denomina fibroma irritativo $(3,5,7,11)$, fibroma traumático $(3,11,13,14,15)$, fibroma oral $(3,5,7,14,15)$, y fibroma fibromatosis (5), aunque el término de fibroma hace referencia a una neoplasia proliferativa benigna de los fibroblastos $(5,14)$, lo cual dista del origen de esta lesión, por ello, se prefiere utilizar el término de hiperplasia fibrosa focal, pues implícitamente lo describe como una respuesta tisular reactiva $(5,7,11)$, al mismo tiempo de que especifica su apariencia clínica y su patogénesis. (7).

La hiperplasia fibrosa focal puede ocurrir en cualquier parte de la cavidad bucal, si bien el sitio predominante es en mucosa yugal a lo largo del plano de oclusión $(2,3,5,7,10,11,13,14,16)$, también puede presentarse en gíngiva $(2,3,5,7,11,14,15)$, lengua (dorso o borde lateral de lengua) $(5,7,10,11,14,16)$, labio $(5,7,10,11,14)$, reborde alveolar edéntulo (11) y paladar $(5,7,11)$, esta última zona es poco común debido a las pocas posibilidades de trauma local.

En la zona posterior es rara su localización, aunque existe un reporte de Lanjekar et al. (2016), que informa de un caso de una lesión en la región posterior mandibular izquierda sobre reborde alveolar (16), asimismo, se demuestra otro caso en la figura1-D de esta revisión, de una lesión en la zona retromolar inferior izquierda, figura que ejemplifica diferentes zonas de aparición de este tipo de lesión.

Las características clínicas de la hiperplasia fibrosa focal se puntualizan en la tabla 1, lo que ayuda a identificar los detalles para realizar el diagnóstico de la lesión. 

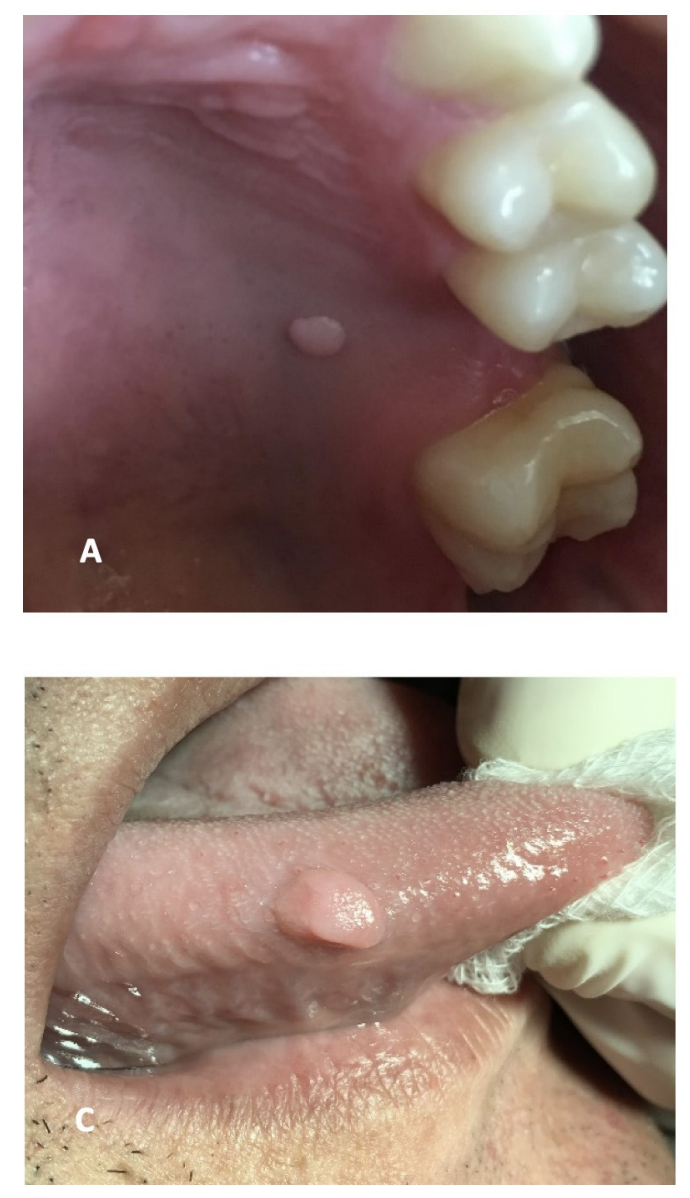

Figura 1. Ejemplos de localización de la lesión hiperplasia fibrosa focal:

A. Mucosa de paladar duro, B. Mucosa de labio inferior, C. Superficie dorsal de lengua,

D. Reborde alveolar, zona retromolar.

\section{Diagnóstico diferencial}

Es importante mencionar que el diagnóstico diferencial puede depender de la zona de localización, a pesar de que es un sobrecrecimiento de tejido suave, con características similares a las estructuras circundantes normales. En esta categoría se deben de considerar:

- Neurofibroma $(7,9)$

- Lipoma $(5,9,10)$

- Hemangioma $(9,10)$ y granuloma piógeno $(9,11)$

- Papiloma $(5,7,9,10)$

- Fibroma periférico osificante $(5,9,11)$, fibroma de células gigantes $(5,7,9,11)$, fibroma odontogénico periférico, tumor odontogénico periférico adenomatoide (9), granuloma gigante de células periféricas $(7,9)$, ameloblastoma periférico (9)
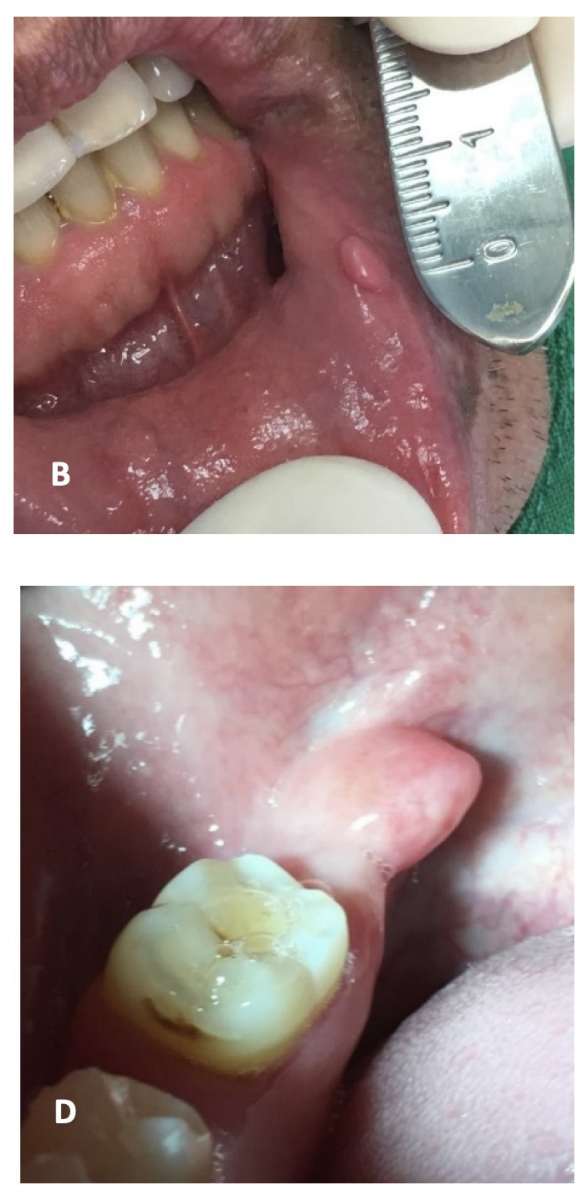
Tabla 1. Características clínicas de la hiperplasia fibrosa focal.

\begin{tabular}{|c|c|}
\hline Característica & Hallazgo específico \\
\hline Sintomatología & $\begin{array}{l}\text { - Asintomático. }(3,5,7,10,11,14,15,16) \\
\text { - El paciente generalmente es consciente de la presencia de la lesión por lo que permanece por un } \\
\text { tiempo prolongado sin tratamiento alguno, a consecuencia de la carencia de síntomas. (7) }\end{array}$ \\
\hline Crecimiento & $\begin{array}{l}\text { - Lento. }(7,9,10,16) \\
\text { - El crecimiento lento se debe al bajo índice mitótico. (10) }\end{array}$ \\
\hline Consistencia & - Suave o dura. $(3,5,10,11,13,16)$ \\
\hline Contorno & - Bien definido y limitado. $(5,7,9,10,11,16)$ \\
\hline Tipo de base & - Sésil o pedunculada. $(3,5,9,10,11,14,15,16)$ \\
\hline Forma & - Redondeada u ovoide. $(3,8)$ \\
\hline Color & $\begin{array}{l}\text { - Similar al tejido circundante. }(3,5,10,11,13,16) \\
\text { - La causa se debe a la reducida vascularidad. }(10,13,16) \text { Sin embargo, puede ser más pálido por la } \\
\text { hiperqueratosis epitelial o, también, estar ulcerada por trauma. }(3,5,11,14,15)\end{array}$ \\
\hline Tamaño & $\begin{array}{l}\text { - Menor a } 20 \text { mm, a pesar de que existen estudios que describen lesiones mayores. }(7,9,10,14,16) \\
\text { - A cualquier edad: }(7,9,10)\end{array}$ \\
\hline Género & $\begin{array}{l}\text { - Proporción doble en mujeres en comparación a varones. }(3,5,7,9,16) \\
\text { Se piensa que las hormonas tienen un papel importante en la etiología. }(3,11,16) \text { En un estudio de } \\
\text { Santana Santos y colaboradores (2014), se señala que la hiperplasia fibrosa focal afecta a población } \\
\text { de todas las edades y a ambos géneros, no obstante, se describe que prevalece el grupo de mujeres } \\
\text { adultas. (11) Igualmente, el estudio de Reddy y colaboradores (2012), indica que estas lesiones se } \\
\text { observan ligeramente más en mujeres. (2) De la misma forma, se sugiere que la predilección en adul- } \\
\text { tos es, probablemente, relacionada a las características físicas en este rango de edad, lo cual incluye } \\
\text { uso de dentaduras, hábito de mordida de labio inferior, mordida crónica de mejilla, entre otros. (20) }\end{array}$ \\
\hline Malignidad & - No. $(10,16)$ \\
\hline
\end{tabular}

epitelio hiperqueratósico $(5,11,14,15)$, la cual puede estar también ulcerada debido al trauma constante $(3,5,11,14,15)$, provocando un exceso de queratina y edema intracelular de las capas superficiales (11), como se observa en la figura 2.

\section{Epidemiología}

La literatura reporta múltiples estudios referentes a la prevalencia de la hiperplasia fibrosa focal en diferentes poblaciones $(1,2,3,4,6,8,11,12,13,17,18$, 19,20), lo cual se visualiza en la tabla 2; además, como se mencionó previamente, las discrepancias en estos, pueden ser debido a los distintos estilos de vida, zonas geográficas, discordancia en la nomenclatura aplicada a estas lesiones, características étnicas, y la población de estudio involucrada, así como si el diagnóstico se realizó solo clínicamente o con estudio histopatológico, lo cual puede afectar el diagnóstico real de la lesión (8).
En múltiples estudios se evidencia que entre las lesiones más habituales en cavidad oral se encuentra la hiperplasia fibrosa focal, en un rango de $4,5 \%$ a $57,4 \%(1,2,3,4,6,8,12,17,19,20)$, aunque en el trabajo de Agrawal y colaboradores (2015) solo se encontró un caso de fibroma en un grupo de 133 lesiones de cavidad oral (18).

La literatura refiere en cuanto al rango de edad de aparición de este tipo de lesión, se encuentra entre 9 y más de 90 años $(6,8,12,19)$.

Asimismo, en los estudios se enfatiza que la lesión tiene predominio en el género femenino $(6,8,11,12,13,17)$, a excepción del trabajo de Kashyap et al. (2012), que alude a que existe mayor afectación en el género masculino (69\%) (3), y el estudio de Vale et al. (2013), el cual subraya la misma proporción con respecto al género (19). 


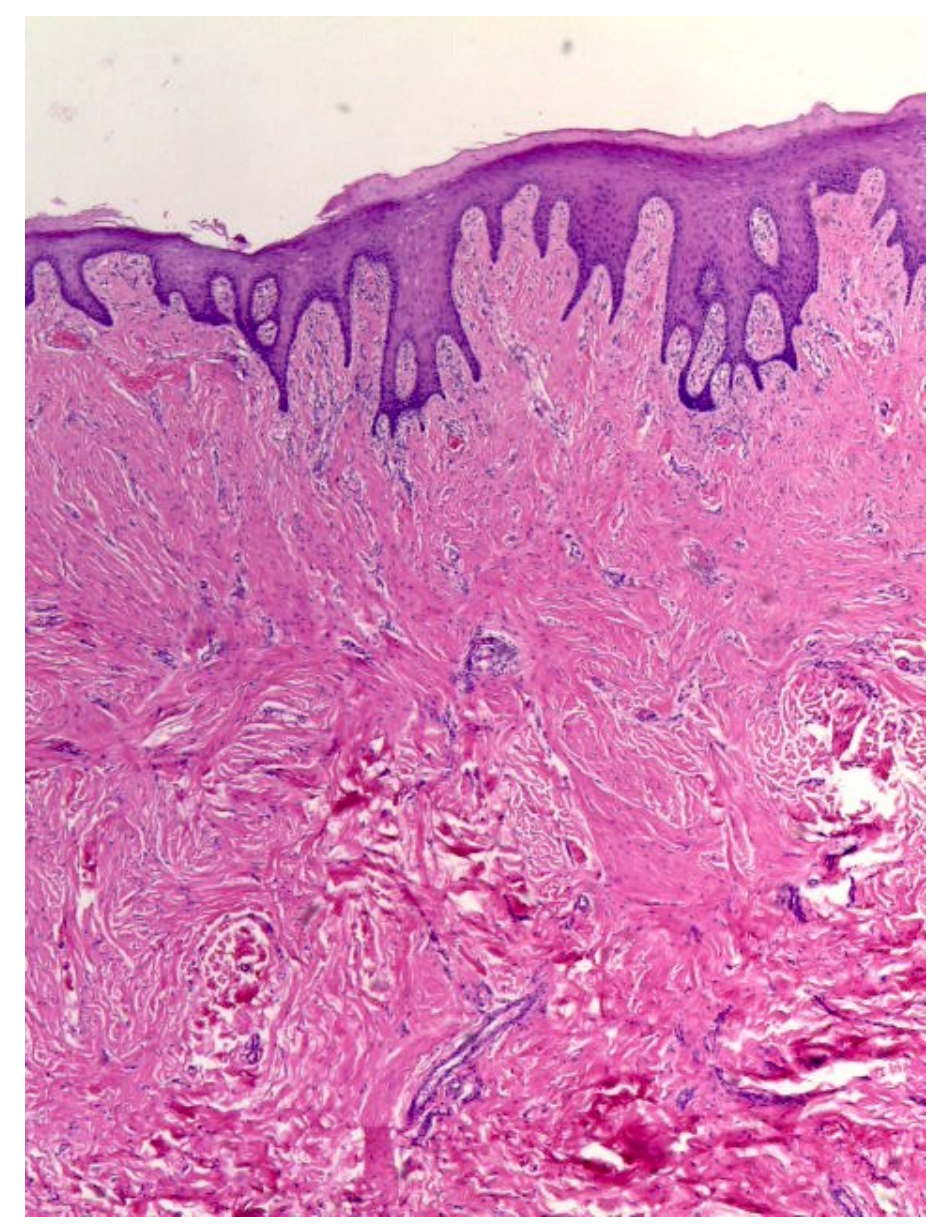

Figura 2. Fotografía de estudio histopatológico de la lesión "hiperplasia fibrosa focal". 20X, tinción hematoxilina-eosina, donde se observa lesión recubierta por epitelio escamoso hiperplásico, sin atipia ni cambios displásicos con presencia de incrementos de colágeno con áreas de hialinización en la lámina propia. Foto de la imagen histológica: cortesía Dr. Diego Guillén Colombari.

La hiperplasia fibrosa focal, según la literatura científica se puede localizar en gíngiva, labio, paladar, lengua, mucosa bucal y mucosa alveolar (1, $2,3,4,6,8,12,13,17)$.

\section{Tratamiento}

El tratamiento de elección es la excisión simple con bisturí $(3,5,8,9,10,11,12,14,15,16)$, sin embargo, también se utiliza laser de dióxido de carbono $(5,10$, 11) y la criocirugía (10); aunque en estos últimos se limita la evidencia para el estudio histopatológico respectivo.

Es importante resaltar que la recidiva es rara, a menos que la remoción sea incompleta o el trauma local continúe $(5,9,10,11,16)$, por lo que es esencial la remoción de la lesión con los irritantes locales que la producen $(3,8,12,16)$, ya que por ejemplo, el estudio de De Santana Santos et al. (2014), basado en revisar 193 casos de hiperplasia fibrosa focal, de forma retrospectiva, destaca que existió un 90,7\% con antecedente de trauma, y que hubo dos casos de recidiva (11).

\section{DISCUSIÓN}

Las lesiones de la mucosa oral son lesiones que producen una alteración de su estructura en cuanto a color, aspecto o pérdida de integridad de la superficie (1), ya que está expuesta a estímulos externos e internos, que pueden provocar desde lesiones de desarrollo, lesiones reactivas e inflamatorias hasta lesiones neoplásicas $(1,2,8)$, interfiriendo con la calidad de vida del paciente $(1,13)$. 
Tabla 2. Estudios de prevalencia de la hiperplasia fibrosa focal y algunas características de la lesión

\begin{tabular}{|c|c|c|c|c|c|}
\hline Autor/año, lugar & $\begin{array}{l}\text { Total de la } \\
\text { muestra del } \\
\text { estudio }\end{array}$ & $\begin{array}{l}\text { Porcentaje de } \\
\text { hiperplasia fibrosa } \\
\text { focal } \\
(\%)\end{array}$ & Lugar predominante & $\begin{array}{l}\text { Rango o } \\
\text { promedio de } \\
\text { edad }\end{array}$ & Género \\
\hline $\begin{array}{l}\text { Hunasgi y colaboradores, } \\
\text { (2020), India. (12) }\end{array}$ & 460 & 10.0 & región anterior inferior & 37.3 años & mujer \\
\hline $\begin{array}{l}\text { Soyele y colaboradores, } \\
\text { (2019) Nigeria. (8) }\end{array}$ & 78 & 39.7 & gíngiva inferior & $\begin{array}{l}\text { 9-29 años } \\
(\text { rango) }\end{array}$ & mujer \\
\hline $\begin{array}{l}\text { Toum y colaboradores, } \\
\text { (2018), Líbano. (1) }\end{array}$ & 178 & 4.5 & --- & --- & mujer \\
\hline $\begin{array}{l}\text { Miyake y colaboradores, } \\
\text { (2018), Tokyo, Japón. (17) }\end{array}$ & 296 & 43.6 & $\begin{array}{l}\text { ápex, borde lateral, } \\
\text { dorso y región ventral } \\
\text { de la lengua }\end{array}$ & $\begin{array}{l}\text { 13-87 años } \\
\text { (rango) }\end{array}$ & mujer \\
\hline $\begin{array}{l}\text { Rivera y colaboradores, } \\
\text { (2017), Chile. (13) }\end{array}$ & 277 & 10.8 & mucosa de mejilla & --- & mujer \\
\hline $\begin{array}{l}\text { Reddy y colaboradores, } \\
(2012), \text { India. }(2)\end{array}$ & 209 & 57.4 & gíngiva & 36.56 años & mujer \\
\hline $\begin{array}{l}\text { Qannam y Bello, (2016), } \\
\text { Arabia Saudí. (6) }\end{array}$ & 2131 & 46.3 & mucosa bucal & 65 años & mujer \\
\hline $\begin{array}{l}\text { Agrawal y colaboradores, } \\
\text { (2015), India. (18) }\end{array}$ & 133 & 0.7 ( 1 caso $)$ & --- & (rango) & hombre \\
\hline $\begin{array}{l}\text { Vale y colaboradores } \\
(2013), \text { Brasil. (19) }\end{array}$ & 315 & 8.86 ( 18 casos $)$ & --- & $\begin{array}{l}4 \text { años } \\
(0-10 \text { años }) \\
14 \text { años } \\
(11-18 \text { años })\end{array}$ & $1: 1 *$ \\
\hline $\begin{array}{l}\text { Kashyap y colaboradores, } \\
(2012), \text { India. (3) }\end{array}$ & 100 & 35 & mucosa bucal & 35 años & hombre \\
\hline $\begin{array}{l}\text { De Santana Santos y } \\
\text { colaboradores, (2014), } \\
\text { Brazil. (11) }\end{array}$ & 1290 & 14.96 & mucosa bucal & 41.4 años & mujer \\
\hline $\begin{array}{l}\text { Mujica y colaboradores, } \\
\text { (2008), Venezuela. }(20)\end{array}$ & 306 & 7.0 & --- & $\begin{array}{l}13-87 \text { años } \\
\text { (rango) }\end{array}$ & mujer \\
\hline $\begin{array}{l}\text { Zarei y colaboradores } \\
\text { (2007), Iran. (4) }\end{array}$ & 172 & 25.58 & gíngiva & 39 años & mujer \\
\hline
\end{tabular}

*Proporción 1:1 hombre - mujer

---: dato no disponible

La hiperplasia fibrosa focal es la lesión reactiva local más común en cavidad oral $(5,7,9,11,13)$, por lo que la escasa literatura existente obliga a realizar esta revisión bibliográfica.

El conocimiento de lesiones comunes en cavidad oral y sus características clínicas, epidemiológicas e histológicas es fundamental, en beneficio de un diagnóstico temprano y un manejo apropiado, ya que se debe descartar lesiones con una apariencia similar a una hiperplasia fibrosa focal que pueda afectar las opciones de tratamiento. Esta revisión permite presentar un tipo de lesión común, como es la hiperplasia fibrosa focal, que frecuentemente no adquiere la importancia correspondiente por los profesionales, pero que es relevante realizar su diagnóstico mediante una detallada historia clínica, descripción exacta de la lesión, en cuanto a tamaño, 
forma, color, evolución y textura, entre otros, obteniendo así una documentación completa de la patología a tratar, y mediante la necesidad de obtener un diagnóstico histológico para confirmación del tipo de lesión, con el objetivo de brindar un manejo adecuado de la patología oral.

Es claro, para finalizar, que la histopatología es el estándar de oro ante toda lesión en cavidad oral para poder determinar el diagnóstico preciso $(9,11,12,15)$.

\section{CONCLUSIONES}

La hiperplasia fibrosa focal es una lesión común en cavidad oral, que frecuentemente no adquiere la importancia correspondiente. Es transcendental reforzar la destreza y habilidad del profesional, en cuanto a distinguir los hallazgos fuera de las condiciones normales de cavidad oral así como poseer los conocimientos claros ante el diagnóstico y manejo adecuado de cualquier tipo de lesión que se presente, para definir la conducta a seguir.

Agradecimiento: Al Dr. Diego Guillén Colombari por su colaboración con el estudio histopatológico y la respectiva imagen.

Conflicto de intereses: Las autoras no tienen conflictos de interés profesional, personal, financiero o de cualquier otra índole que pudieran influir en el contenido del trabajo.

\section{Correspondencia:}

María del Carmen Navas-Aparicio

Apartado 4841-1000 San José, Costa Rica

Correo electrónico: mcnavasaparicio@hotmail.com

\section{REFERENCIAS BIBLIOGRÁFICAS}

1. Toum SE, Cassia A, Bouchi N, Kassab I. Prevalence and distribution of oral mucosal lesions by sex and age categories: a retrospective study of patients attending lebanese School of Dentistry. Int J Dent.2018; 2018 (4): 1-6. Doi: 10.1155/2018/4030134

2. Reddy V, Saxena S, Saxena S, Reddy M. Reactive hyperplastic lesions of the oral cavity: a ten year observational study on North Indian population. J Clin Exp Dent. 2012;4(3): e136 - 40. Doi: 10.4317/ jced.50670

3. Kashyap B, Reddy PS, Nalini P. Reactive lesions of oral cavity: a survey of 100 cases in Eluru, West Godavari district. Contemp Clin Dent. 2012; 3: 294 7. Doi: 10.4103/0976-237X.103621

4. Zarei RM, Chamani G, Amanpoor S. Reactive hyperplasia of the oral cavity in Kerman province, Iran: a review of 172 cases. Br J Oral Maxillofac Surg. 2007; 45: 288 - 92. Doi: 10.1016/j.bjoms.2006.10.001

5. Ayekinam K, Harti Karima E, Wady Wafaa E. Surgical removal of a focal fibrous hyperplasia: Two case reports. Int J Appl Dent Sci. 2017; 3(2): 215-7.

6. Qannam A, Bello IO. The range of diagnoses for oral soft-tissue biopsies of geriatric patients in a Saudi Arabian teaching hospital. Saudi Dent J. 2016, 28: 96 - 101. Doi: 10.1016/j.sdentj.2016.01.001

7. Mathur LK, Bhalodi AP, Manohar B, Bhatia A, Raí N, Mathur A. Focal fibrous hyperplasia: a case report. Int J Dent Clin. 2010; 2(4): 56 - 7.

8. Soyele OO, Ladeji AM, Adebiyi KE, Adesina OM, Aborisade AO, Olatunji AS, Adeola HA. Pattern of distribution of reactive localized hyperplasia of the oral cavity in patients at a tertiary health institution in Nigeria. Afri Health Sci. 2019; 19(1): 1687 - 94. Doi:.4314/ahs.v19i1.45

9. Vergotine RJ. A giant cell fibroma and focal fibrous hyperplasia in a young child: a case report. Case Rep Dent. 2012; 2012: 370242. Doi: 10.1155/2012/370242

10. Valério RA, Mussolino de Queiroz A, CoutinhoRomualdo P. Brentegani LG, Garcia de Paula-Silva FW. Mucocele and fibroma: treatment and clinical features for differential diagnosis. Braz Dent J. 2013; 24(5): 537 - 41 Doi: 10.1590/0103-6440201301838

11. de Santana Santos T, Martins-Filho PS, Piva MR, de Souza Andrade ES. Focal fibrous hyperplasia: A review of 193 cases. J Oral Maxillofac Pathol. 2014; 18: 86 - 9. Doi: 10.4103/0973-029X.141328

12. Hunasgi S, Koneru A, Vanishree M, Manvikar V. Assessment of reactive gingival lesions of oral cavity: A histopathological study. J Oral Maxillofac Pathol. 2017; 21:180. Doi: 10.4103/jomfp.JOMFP_23_16

13. Rivera C, Droguett D, Arenas-Márquez MJ. Oral mucosal lesions in a Chilean elderly population: A retrospective study with a systematic review from thirteen countries. J Clin Exp Dent. 2017; 9(2): e276 83. Doi: $10.4317 /$ jced. 53427

14. Regezi J, Sciubba J, Jordan R. Oral Pathology: Clinical Pathologic Correlations. 6 ed. St Louis Missouri: Elsevier; 2012. p. 407.

15. Saap JP, Eversole LR, Wysocki GP. Patología Oral y Maxilofacial Contemporánea. España: Harcourt; 1998. p. 33.

16. Lanjekar A, Kulkarni S, Akhade S, Sonule S, Rathod U. An unusually large irritation fibroma associated with gingiva of lower left posterior teeth region. Case Rep Dent. 2016; 2016: Doi: 10.1155/2016/5202181

17. Miyake Y, Shinozuka K, Ueki K, Teraoka J, Zama M, Ogisawa S, Shinozaki Y, Aoki J, Yanagawa K, 
Shimizu O, Kaneko T, Tonogi M, Onki H. Retrospective clinical study of 296 patients with mass lesions of the tongue. J Oral Sci. 2018; 60 (4): 574-8. Doi: 10.2334/ josnusd.17-0317

18. Agrawal R, Chauhan A, Kumar P. Spectrum of oral lesions in a tertiary care hospital. J Clin Diagn Res. 2015; 9(6): EC11 - 3. Doi: 10.7860/jcdr/2015/ 13363.6121

19. Vale EB, Ramos-Perez FM, Rodrigues GL, Carvalho EJ, Castro JF, Perez DE. A review of oral biopsies in children and adolescents: a clinicopathological study of a case series. J Clin Exp Dent. 2013;5(3): e144 - 9. Doi: 10.4317 /jced.51122

20. Mujica V, Rivera H, Carrero M. Prevalence of oral soft tissue lesions in an elderly venezuelan population. Med Oral Patol Oral Cir Bucal. 2008;13(5):270 -4.

Recibido : 08-02-2021

Aceptado : 22-04-2021 